This document is the Accepted Manuscript version of a Published Work that appeared in final form in The Journal of Physical Chemistry A, copyright (C) American Chemical Society after peer review and technical editing by the publisher. To access the final edited and published work see https://pubs.acs.org/doi/10.1021/acs.jpca.6b02230. 


\section{Structure and Stability Studies of Pharmacologically Relevant $S$ - nitrosothiols: A Theoretical Approach}

Benjamin Meyer ${ }^{(1,2)}$, Alessandro Genoni ${ }^{(1,2) *}$, Ariane Boudier ${ }^{(3)}$, Pierre Leroy ${ }^{(3)}$, Manuel F. Ruiz-Lopez ${ }^{(1,2) *}$

(1) CNRS, Laboratoire SRSMC, UMR 7565, Vandoeuvre-lès-Nancy, F-54506, France.

(2) Université de Lorraine, Laboratoire SRSMC, UMR 7565, Vandoeuvre-lès-Nancy, F54506, France.

(3) Université de Lorraine, Laboratoire CITHÉFOR, EA 3452, Nancy, F-54001, France.

* Correspondence to:

- Alessandro Genoni, CNRS and Université de Lorraine, Laboratoire SRSMC - UMR 7565, Boulevard des Aiguillettes, BP 70239, 54506 Vandoeuvre-lès-Nancy, France. E-mail: Alessandro.Genoni@univ-lorraine.fr; Phone : +33 (0)3 836843 77; Fax: +33 (0)3 836843 71.

- Manuel F. Ruiz-Lopez, CNRS and Université de Lorraine, Laboratoire SRSMC - UMR 7565, Boulevard des Aiguillettes, BP 70239, 54506 Vandoeuvre-lès-Nancy, France. E-mail: Manuel.Ruiz@univ-lorraine.fr; Phone : +33 (0)3 836843 78; Fax: +33 (0)3 83684371. 


\begin{abstract}
Nowadays, $S$-nitrosothiols (RSNOs) represent a promising class of nitric oxide (NO) donors that could be successfully used as drugs to compensate the decrease of NO production that usually arises in conjunction with cardiovascular diseases. Nevertheless, notwithstanding their pharmacological interest, the structure-stability relationship in RSNOs is still unclear and this issue, together with the mechanism of NO donation in the physiological medium, deserves further investigation. As a first step forward in this direction, in this paper, the overall stability and structural preference of two pharmacologically relevant $S$-nitrosothiol molecules have been studied in detail by means of computational strategies. In particular, performing calculations in implicit solvent (water) on the $S$-nitroso- $N$-acetylpenicillamine (SNAP) and the $S$-nitroso- $N$-acetylcysteine (NACNO) and analyzing the non-covalent interactions networks of their most stable conformers, we have observed that the structure and the stability of these molecules can be directly related to the formation of stabilizing hydrogen-bond and chalcogen-chalcogen intra-molecular interactions.

The obtained results represent the starting point for further investigations to be conducted also on larger RSNOs in order to shed further light on the role played by intra- and inter-molecular interactions and by solvation effects in stabilizing this class of molecules. The obtained insights will be hopefully helpful to design new RSNO-based drugs characterized by an enhanced pharmacological potency.
\end{abstract}




\section{Introduction}

Nitric oxide (NO), the neutral +2 oxide of nitrogen, is a gaseous transmitter with a short halflife (within one second) that exhibits many physiological functions in the cardiovascular system and that especially maintains the homeostasis of the vessel tone. Nevertheless, the NO production in the body decreases during ageing with a concomitant increase of cardiovascular diseases (CVDs), such as atherosclerosis, angina pectoris (angor) and stroke. ${ }^{1}$ Therefore, NO donors have been introduced in therapeutics for a long time, mainly as organic nitrates. However, they present major drawbacks (tolerance phenomenon, oxidative stress induction) that limit their use mostly to acute events in CVDs and preclude their extensive use in chronic treatments. $^{2}$ Thus, other classes of NO donors have been investigated and the $S$-nitrosothiols (RSNOs) have emerged as promising drug candidates since they are not characterized by the typical disadvantages associated with organic nitrates ${ }^{3}$ and since they generally exhibit much longer half-lives. Among them it is worth mentioning the $S$-nitrosoglutathione (GSNO), a major physiological RSNO that has been extensively used in both preclinical and clinical studies ${ }^{4}$, and the $S$-nitroso- $N$-acetylpenicillamine (SNAP) and the $S$-nitroso- $N$-acetylcysteine (NACNO), which have been particularly reported for their pharmacological interest (see Chart 1).<smiles>NC(CCC(=O)NC(CSN=O)C(=O)NCC(=O)O)C(=O)O</smiles><smiles>CC(=O)NC(C(=O)O)C(C)(C)SN=O</smiles>

SNAP<smiles>CC(=O)NC(CSN=O)C(=O)O</smiles>

NACNO

Chart 1

Unfortunately, there is currently a lack of knowledge concerning the relationship between the RSNOs physicochemical properties and their bioactivity and stability, which can be related to 
the difficulty for the RSNOs to dissociate into the corresponding RS• and NO• radicals. In this context it is worthwhile to note that the RSNOs usually exhibit a considerable S-N double bond character and, consequently, two geometrical isomers are possible ${ }^{3}$, syn and anti (see Chart 2), with values of Bond Dissociation Energies (BDEs) reported in the range of 20-31 $\mathrm{kcal} / \mathrm{mol}^{5}$ Therefore, it is obvious that the RSNOs stability depends on their structure. Furthermore, two main kinds of RSNOs exist and they mainly differ for the number of substituents on the carbon atom located at the $\alpha$-position of the S-NO group. They are tertiary and primary RSNOs, such as SNAP and NACNO, respectively.
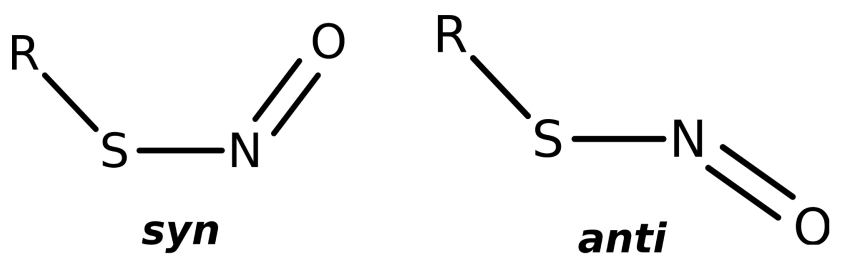

Chart 2

Among all the RSNOs, NACNO has been observed as the most stable in solution, with halflife time of $500 \mathrm{~h}^{3}$ In comparison, the half-life time of SNAP is $2 \mathrm{~h}^{3}$ This relative stability between SNAP and NACNO contrasts with the conclusions of some previous studies that concluded that tertiary RSNOs should be more stable than the primary ones. ${ }^{6}$ Indeed, controversies about the relative stabilities of the RSNO compounds and about the releasemechanisms of NO species still exist in the literature, although the mechanism of S-NO bond cleavage seems to occur through a homolytic rather than a heterolytic process, liberating the neutral NO• radical and involving the formation of a disulfide.

A better understanding of how the stability of RSNO compounds in aqueous media is related to their chemical structure is therefore clearly needed in view of developing novel drug candidates for therapeutic uses. Actually, this issue has already motivated a number of theoretical works that have tried to clarify the chemical properties of the SNO function, 
providing estimations for the BDEs of several model systems ${ }^{7-26}$. Calculations and experiments reported by Bartberger et al. ${ }^{7,8}$ have provided a new consistent picture of the conformational behavior of the RSNOs. Their results suggest a competing steric effect that favors the anti orientation of the RSNO group when the R group is large. At the same time, Lü et $a l .{ }^{9}$ have proposed an experimental and theoretical approach to calculate the S-NO bond heterolysis and homolysis energies using a thermodynamic cycle. Moreover, since different theoretical investigations had provided $\mathrm{S}-\mathrm{N}$ bond lengths and BDEs that fluctuate considerably, Baciu and Gauld ${ }^{10}$ have reported an assessment of the usually employed theoretical methods to obtain accurate structures and homolytic BDEs. Some benchmark studies on $\mathrm{CH}_{3} \mathrm{SNO}$ were also reported by Fu et al. ${ }^{11}$ The nature of the S-NO bond in RSNOs has been investigated by Timerghazin et al. ${ }^{12}$ who have proposed a representation of the SNO group in terms of three different resonance contributions: a conventional (single bond), a zwitterionic and an ionic form, as represented in Chart 3. In order to directly take into account the potential multireference character of the RSNOs wave functions associated with these three resonance structures, Timerghazin and coworkers performed the first multiconfigurational calculations on the simple HSNO molecule using the CASSCF and CASPT2 methods. ${ }^{13}$

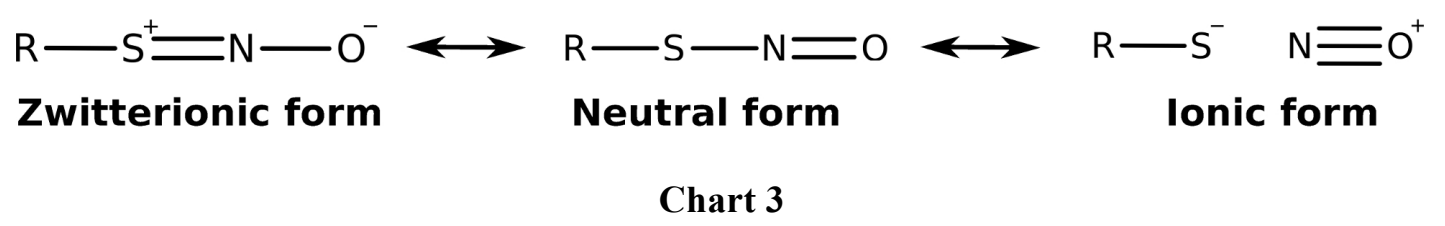

Other studies have investigated more complex RSNOs and/or reaction mechanisms. For instance, it is worth mentioning the research on the factors regulating the structure and the stability of the aromatic RSNOs ${ }^{14}$ or the studies conducted on series of nitrosothiolderivatives to investigate substituents and solvent effects on the BDEs. ${ }^{11,15}$ Moreover, it is 
important to consider the fundamental studies conducted by Talipov and Timerghazin, who have interestingly shown through proper computational models that, in enzymatic processes, the RSNOs structure, stability and reactivity can be significantly modified by interactions with charged and polar residues in proteins ${ }^{16}$ and by the influence of biologically accessible external electric fields. ${ }^{17}$ Finally, for the sake of completeness, we can also mention the works carried out by Marazzi and coworkers, in which the focus shifted to the photo-chemistry and the photo-physics of a series of $\operatorname{RSNOs}^{21,22}$ and in which the photochemical rupture of the S$\mathrm{N}$ bond has been studied in detail.

However, despite all these investigations, the electronic properties of RSNOs remain an open controversy. The rather high rotation barriers observed between the syn and the anti conformations imply a partial S-N double bond character (see Chart 3), while the small homolytic BDEs $(\sim 30 \mathrm{kcal} / \mathrm{mol})$ and the elongated $(1.8 \AA) \mathrm{S}-\mathrm{N}$ bond lengths suggest that this bond is relatively weak ${ }^{10-14}$. In addition, environmental effects are presumably important because polar media should favor the zwitterionic and ionic forms of the bond, but this issue has not been investigated yet.

In this paper, we report a detailed theoretical study on the pharmacological relevant molecules SNAP and NACNO. These systems are particularly interesting from the theoretical point of view because they display a significant stability difference despite their close chemical structure (see Chart 1; they differ by the presence of two methyl groups on the carbon- $\alpha$ in SNAP instead of two hydrogen atoms in NACNO). At first, exploiting quantum chemistry methods, we will present a thorough conformational analysis of the systems under exam, which, up to now, has not been reported in the literature, although it is fundamental in order to understand the relative stability of these compounds. Moreover, in our study, we will take into account the role of the aqueous solvent, which has been neglected in most of the previous theoretical investigations on the RSNO derivatives. In fact, solvent effects are expected to 
play a major role in this case because both SNAP and NACNO (see Chart 1) bear carboxylic acid groups that should be ionized at the typical $\mathrm{pH}$ of physiological media. Furthermore, the interactions of the carboxylate moiety with the SNO function in the molecules, and/or with the unpaired electron of the $\mathrm{S} \cdot$ center in the radicals, might substantially influence the dissociation properties of the S-NO bond, but this aspect has not been analyzed so far.

For all these reasons, we expect that the ensemble of results obtained in this work could become useful in the design of new RSNO molecules with higher therapeutic interest. In particular, we believe that the analysis of the intra-molecular interactions in the examined compounds could be of paramount importance to model phamacophores for the proposition of alternative RSNO-based drugs characterized by longer half-lives.

We want to stress that the goal of this paper is to start investigating the reasons for the different chemico-physical stabilities of pharmacologically relevant RSNO compounds (especially SNAP and NACNO). This study is crucial because, first of all, to be an efficient NO-donor drug, a molecule must deliver the NO radical over a sufficiently long lapse of time. Without the fulfillment of this condition, which represents one of the first screenings in the search of novel NO-donor drugs, a compound is not even considered. Of course, to complete the drug discovery process, the next step will consist in taking into account the interactions of the selected compounds with the biological environment, also in light of recent studies conducted by Timerghazin and coworkers ${ }^{16,17}$, who pointed out how structure, stability and reactivity of the RSNOs could dramatically change due to the interactions with protein residues.

The paper is organized as follows. At first, we will describe the methods that we have used to investigate the structure and the stability of all the considered molecules. Then, in the following section, after showing the results obtained for the small model compounds $\mathrm{CH}_{3} \mathrm{SNO}$ and $\left(\mathrm{CH}_{3}\right)_{3} \mathrm{CSNO}$, we will focus on the different calculations and analyses 
performed to study the stability of SNAP and NACNO. Particular attention will be devoted to the performed conformational analyses and to the interpretation of the different molecular stabilities in terms of the intra-molecular interactions in the investigated compounds. Finally, some conclusions and perspectives will be presented.

\section{Computational Details}

In this section we will provide the details of the computational techniques that we have exploited to study the stability of the RSNO systems. We point out that, if not stated otherwise, all the quantum chemistry computations in this paper have been carried out using the quantum chemistry package Gaussian $09 .{ }^{27}$

As in previous studies (see the Introduction), the stability of the RSNO compounds will be discussed by inspecting the S-N bond dissociation energies (BDE). However, one might wonder whether or not the dissociation process involves an activation energy barrier, which would control the reaction kinetics. The answer to this question is not easy. On the one hand, Houk and coworkers ${ }^{8}$ reported calculations for the model compound $\mathrm{CH}_{3} \mathrm{SNO}$ in gas phase. The potential energy surface (CBS-QB3 ab initio level) did not display any saddle point (this fact was not explicitly discussed but it can be deduced from the enthalpy values reported in

Table 1 of the paper ${ }^{8}$ ). However, using the Variational Transition State Theory, the authors predicted the existence of a free energy barrier at a quite long S-N distance (3.55 $\AA$ ). In the liquid phase, to our knowledge, no systematic study of the dissociation energy surface has been reported and it has been generally assumed that the RSNOs stability is related to the homolytic BDE (see, for instance, the work by Lü et al. ${ }^{9}$ where a comparison of heterolytic and homolytic dissociation energies in acetonitrile was analyzed). This assumption seems chemically reasonably because if an activation free energy barrier exists at such long S-N distances (late transition state), its height should strongly depend on the BDE. On the other 
hand, technically, it is extremely problematic to locate such a hypothetical activation barrier because a) the quantum mechanical method must be able to (quantitatively) deal with the open shell singlet in RS $\cdots \mathrm{NO}$ structures, and this may require exploiting multireference approaches; b) the use of an explicit solvent model becomes compulsory in that case. The computational cost of such a study is prohibitive and, therefore, following other studies, the relative stability of RSNO compounds will be assessed through the use of the homolytic BDE. As usual, in our investigation, the theoretical level was chosen trying to get a good compromise between accuracy and computational cost. In particular, in the present study we have faced the need of exploring in detail the potential energy surfaces of SNAP and NACNO, as well as those of their radicals, which requires several hundreds of energy computations and geometry optimizations. Therefore, it was not possible to carry out very high-level ab initio calculations. However, in previous investigations it has been shown that, for some RSNO properties, DFT (Density Functional Theory) methods generally provide reasonably good agreements with benchmark $a b$ initio calculations ${ }^{8}$ or even experimental data $^{7,8,15}$. In particular, Li et al. ${ }^{15}$ have recently shown that the B3PW91 functional gives suitable BDEs for SNAP-two peptide molecules. Here, Table 1 summarizes several calculations for the energetics of the S-N bond dissociation in the case of the simple model system $\mathrm{CH}_{3} \mathrm{SNO}$ (in syn conformation). In particular, the results obtained exploiting four density functionals (B3LYP ${ }^{28,29}, \mathrm{PBEPBE}^{30}, \mathrm{BVP}^{31}$ and $\mathrm{B} 3 \mathrm{PW} 91^{32}$ ) are compared to the outcomes of MP2 and CBS-QB3 calculations. Overall, the comparison confirms that the B3PW91 functional, combined with the aug-cc-pVTZ basis-set, or even with the smaller 6$311+\mathrm{G}(\mathrm{d}, \mathrm{p})$ basis-set, provides satisfactory results. Hence, in the calculations below, the B3PW91/6-311+G(d,p) theoretical level has been adopted. In this regard it is worth noting that our basis-set is larger than the one used by Li et al. ${ }^{15}(6-31 \mathrm{G}(\mathrm{d}, \mathrm{p}))$ and, above all, that the additional flexibility provided by the use of a triple- $\zeta$ split valence basis and by the inclusion 
of a diffuse function on heavy atoms was considered of crucial importance in view of the study of charged systems in polar media.

Table 1. Energetics of the homolytic S-NO bond dissociation in syn- $\mathrm{CH}_{3} \mathrm{SNO}\left(\mathrm{kcal} \cdot \mathrm{mol}^{-1}\right)$. Comparison of different theoretical approaches. The minimum-energy geometry and the vibrational frequencies of the molecule have been calculated at each computational level.

\begin{tabular}{lccc}
\hline Method & $\Delta \mathrm{E}$ & $\Delta \mathrm{H}(0 \mathrm{~K})$ & $\Delta \mathrm{G}(298 \mathrm{~K})$ \\
\hline CBS-QB3 $^{\mathrm{a}}$ & 34.0 & 31.6 & 21.5 \\
MP2/aug-cc-pVTZ & 35.9 & 35.4 & 25.4 \\
PBEPBE/aug-cc-pVTZ & 44.9 & 42.0 & 31.8 \\
BVP86/aug-cc-pVTZ & 42.2 & 39.3 & 29.2 \\
B3LYP/aug-cc-pVTZ & 31.3 & 28.4 & 18.4 \\
B3PW91/aug-cc-pVTZ & 33.5 & 30.6 & 20.5 \\
B3PW91/6-311+G(d,p) & 32.0 & 29.1 & 19.0
\end{tabular}

As mentioned above, for comparison purposes, we have initially investigated the structure and the S-NO dissociation energies of the $\mathrm{CH}_{3} \mathrm{SNO}$ and $\left(\mathrm{CH}_{3}\right)_{3} \mathrm{CSNO}$ molecules. Their syn and anti conformations have been fully optimized at the B3PW91/6-311+G(d,p) level, as well as the geometries of the associated radicals $\left(\left(\mathrm{CH}_{3}\right)_{3} \mathrm{CS} \bullet, \mathrm{CH}_{3} \mathrm{~S} \bullet\right.$ and $\left.\mathrm{NO} \bullet\right)$. All the obtained structures have been properly characterized as energy minima checking the absence of imaginary vibration frequencies. The zero-point vibrational energies and thermal corrections to Gibbs free energy at $298.15 \mathrm{~K}$ have been computed using the ideal gas and harmonic approximations as implemented in Gaussian09. The obtained Gibbs free energies, which have been calculated assuming a reference state that corresponds to a pressure of $1 \mathrm{~atm}$, have been exploited to evaluate the relative stability of the syn and anti conformers for the two RSNOs and to determine their equilibrium population according to the Boltzmann distribution:

$$
\frac{N_{x}}{N_{T}}=\frac{e^{-\Delta G_{x} / R T}}{\sum_{i=1}^{n_{c}} e^{-\Delta G_{i} / R T}}
$$


where $N_{x} / N_{T}$ is the fraction of molecules that are in the conformation $x$ at the equilibrium, $n_{c}$ is the total number of possible conformers for the system in exam (syn and anti in this case), $\Delta G_{i}$ is the free-energy of the $i$-th conformer relative to the one of the minimum free-energy conformation, $R$ is the molar ideal gas constant and $T$ is the temperature in kelvins.

Other than in gas phase, the systems have been also studied in aqueous media to take into account the physiological/environmental conditions at which the RSNOs physiologically act. The investigation in solution has been conducted using an implicit solvation model, (specifically the IEF-PCM method $^{33-36}$ that assumes the dielectric constant $\varepsilon_{r}=78.3553$ for liquid water) and considering a reference state corresponding to a $1 \mathrm{M}$ concentration. As shown by Kelly et al. ${ }^{37}$, the relation between free energies corresponding to $1 \mathrm{~atm}$ and $1 \mathrm{M}$ reference states is given by

$$
\mathrm{G}(1 \mathrm{M})=\mathrm{G}(1 \mathrm{~atm})+\mathrm{RT} \ln (24.46)
$$

with the correction factor that, at $298 \mathrm{~K}$, amounts to $1.9 \mathrm{kcal} \cdot \mathrm{mol}^{-1}$. Afterwards, a similar study has been carried out for SNAP and NACNO. However, in this case, the study has only been carried out in aqueous solution because the systems bear carboxylic groups, which we assume to be ionized at physiological $\mathrm{pH}$. Furthermore, considering the complexity of the molecules, it appeared necessary to carry out a detailed conformational analysis. A systematic search of energy minima in the potential energy surface was therefore performed, as explained in detail below.

To better explore the reasons for the calculated stabilities of the two systems in exam and of the associated radicals, a comparison of their intra-molecular interactions networks has been also performed using the Non-Covalent Interaction (NCI) methodology. ${ }^{38,39}$ This theoretical approach entirely relies on the analysis of the electron density and of its corresponding reduced gradient, and it has been already successfully used to detect both intra- and intermolecular interactions. These interactions correspond to low-value peaks of the reduced 
density gradient occurring at low values of the electron density and they can be easily visualized in real space plotting proper low-value reduced gradient isosurfaces. For the sake of completeness, all the NCI calculations have been carried out exploiting the software NCIPLOT-3.0 $0^{38,39}$ and using the electron densities resulting from the quantum mechanical computations at B3PW91/6-311+G(d,p) level. More specific details about the NCI computations are provided in the Supporting Information.

Finally, to further investigate the stability of the radicals resulting from the homolytic dissociation of the S-NO bond in SNAP and NACNO, QTAIM ${ }^{40}$ (Quantum Theory of Atoms in Molecules) analyses have been also performed using the AIMAll package ${ }^{41}$ (Version 13.11.04) and mainly focusing on the study of properties directly related to the detected intramolecular non-covalent bond critical points (BCPs).

\section{Results and discussion}

Model systems: $\mathrm{CH}_{3} \mathrm{SNO}$ vs. $\left(\mathrm{CH}_{3}\right)_{3} \mathrm{CSNO}$. To start investigating the role of the substitution of the carbon- $\alpha$ in the stability of the RSNOs, we have studied and compared the conformational and energetic features of the small $\mathrm{CH}_{3} \mathrm{SNO}$ and $\left(\mathrm{CH}_{3}\right)_{3} \mathrm{CSNO}$ molecules. The result of this analysis is shown in Table 2. Provisionally focusing only on the gas phase results, the computation of the free energies at $298.15 \mathrm{~K}$ has revealed that the most stable (and therefore the most highly populated) conformations about the S-N bond for the $\mathrm{CH}_{3} \mathrm{SNO}$ and $\left(\mathrm{CH}_{3}\right)_{3} \mathrm{CSNO}$ systems are syn and anti, respectively. The difference is especially significant in the case of $\left(\mathrm{CH}_{3}\right)_{3} \mathrm{CSNO}$, for which the populations of the anti and syn conformers are approximately in a 12:1 ratio. The obtained results are in agreement with the outcomes of other gas phase calculations, such as the ones reported by Bartberger and coworkers ${ }^{7,8}$ in their pioneering investigations about the conformational behavior and the decomposition rates of the RSNOs and, more recently, by Marazzi et al., ${ }^{22}$ who have computed the energies of the 
syn conformers relative to the ones of the corresponding anti conformers for a large group of quite small RSNO systems. Previous works have generally considered that the preferred anti form in the tertiary RSNO derivatives is a consequence of destabilizing steric interactions in the syn arrangement, even if we will show below that this is perhaps a too simplistic interpretation.

Table 2. Energetics $\left(\mathrm{kcal} \cdot \mathrm{mol}^{-1}\right)$ and fractional equilibrium population (\%) at $298.15 \mathrm{~K}$ for the syn and the anti conformers of the $\mathrm{CH}_{3} \mathrm{SNO}$ and the $\left(\mathrm{CH}_{3}\right)_{3} \mathrm{CSNO}$ molecules.

\begin{tabular}{|c|c|c|c|c|c|c|}
\hline \multirow[b]{2}{*}{ Molecule } & \multicolumn{4}{|c|}{ Gas-Phase } & \multicolumn{2}{|c|}{ Water } \\
\hline & $E_{r e l}$ & $H_{r e l}(0 k)$ & $\Delta G_{r e l}$ & $\begin{array}{l}\text { Equilibrium } \\
\text { Population }\end{array}$ & $\Delta G_{r e l}$ & $\begin{array}{l}\text { Equilibrium } \\
\text { Population }\end{array}$ \\
\hline \multicolumn{7}{|l|}{$\mathrm{CH}_{3} \mathrm{SNO}$} \\
\hline syn & 0.0 & 0.0 & 0.0 & 77.52 & 0.0 & 81.42 \\
\hline $\begin{array}{l}\text { anti } \\
\left(\mathrm{CH}_{3}\right)_{3} \mathrm{CS}\end{array}$ & 0.7 & 0.7 & 0.7 & 22.48 & 0.9 & 18.58 \\
\hline syn & 1.3 & 1.3 & 1.5 & 7.77 & 1.4 & 8.08 \\
\hline anti & 0.0 & 0.0 & 0.0 & 92.23 & 0.0 & 91.92 \\
\hline
\end{tabular}

Afterwards, we have determined the energetics for the S-NO bond dissociation for both the two investigated systems. Only the preferred and most stable conformation has been considered in each case. The results (see Table 3) reveal a higher stability (namely, a higher energy for dissociation into the corresponding $\mathrm{RS} \cdot$ and $\mathrm{NO}$ - radicals) for $\mathrm{CH}_{3} \mathrm{SNO}$, the molecule with the least substituted carbon in $\alpha$ position with respect to the nitrosothiol group. The extra stabilization coming from an intra-molecular $\mathrm{C}-\mathrm{H} \cdots \mathrm{O}$ hydrogen bond in the $\mathrm{CH}_{3} \mathrm{SNO}$ derivative (Chart 4), which is not possible in the tertiary $\left(\mathrm{CH}_{3}\right)_{3} \mathrm{CSNO}$ one, seems to be at the origin of this higher dissociation energy. A more detailed analysis will be presented below for the pharmacologically relevant molecules.

Interestingly, from the values in Tables $2-3$, we can easily note that the introduction of aqueous solvation effects does not essentially change the results obtained from gas phase 
calculations for these small RSNO derivatives. The most noticeable consequence is the increase in relative stability (from 0.7 to $0.9 \mathrm{kcal} \cdot \mathrm{mol}^{-1}$ ) of the $s y n$ conformer with respect to the corresponding anti one for the $\mathrm{CH}_{3} \mathrm{SNO}$ molecule, which entails a slight increase of the syn:anti equilibrium population ratio (from about 3.4 to 4.4 ). The homolytic bond dissociation free energy $\left(\Delta G_{H B D}\right)$ undergoes only very small changes for both the systems, being the difference between the free energy in gas phase and the free energy in water essentially due to the change of the reference state.

Table 3. Homolytic bond dissociation (HBD) energies, enthalpies at $0 \mathrm{~K}$ and free energies at $298.15 \mathrm{~K}$ (in $\mathrm{kcal} \cdot \mathrm{mol}^{-1}$ ) for the S-NO bond in the most stable conformers of the $\mathrm{CH}_{3} \mathrm{SNO}$ and $\left(\mathrm{CH}_{3}\right)_{3} \mathrm{CSNO}$ molecules. Note that gas phase free energies refer to the $1 \mathrm{~atm}$ standard state, while the water solution values refer to the $1 \mathrm{M}$ standard state; according to equation (2), a factor of $1.9 \mathrm{kcal} \cdot \mathrm{mol}^{-1}$ must be added to convert $\Delta G_{H B D}(1 \mathrm{~atm})$ into $\Delta G_{H B D}(1 \mathrm{M})$.

\begin{tabular}{lccccc}
\hline \multirow{2}{*}{$\mathrm{RSNO} \rightarrow \mathrm{RS} \cdot+\mathrm{NO} \cdot$} & \multicolumn{3}{c}{ Gas-Phase } & & Water \\
\cline { 2 - 4 } & $\Delta E_{H B D}$ & $\Delta H_{H B D}(0 K)$ & $\Delta G_{H B D}$ & & $\Delta G_{H B D}$ \\
\hline syn- $\mathbf{C H}$ SNO & 32.0 & 29.1 & 19.0 & 20.7 \\
anti- $\left(\mathbf{C H}_{3}\right)_{3} \mathbf{C S N O}$ & 29.9 & 27.7 & 17.0 & & 18.8 \\
\hline
\end{tabular}

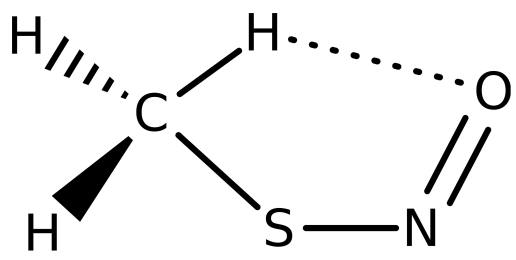

Chart 4

Structure and stability of SNAP and NACNO. The main goal of this investigation was to theoretically determine the homolytic Bond Dissociation Free Energy for the closely related 
compounds SNAP and NACNO with the aim of shedding further light on the role played by the substituents at the carbon- $\alpha$ in the stability of the RSNO molecules. As mentioned above, in aqueous media at physiological $\mathrm{pH}(7.4)$, the carboxylic groups borne by these systems should be ionized and accordingly, in our computations, the two systems were considered as anions. Moreover, to reach our goal and due to the large number of possible intra-molecular interactions in SNAP and NACNO (and in the corresponding RS• radicals), a thorough conformational sampling was necessary in order to identify all the energy minima in the potential energy surface. The strategies adopted for the conformational samplings are described in detail hereafter. All the calculations were carried out at the B3PW91/6$311+\mathrm{G}(\mathrm{d}, \mathrm{p})$ level.

Concerning the undissociated systems, at first, their geometry has been fully optimized starting from an arbitrary conformation chosen on the basis of chemical intuition. Afterwards, starting from this initial optimized geometry, we have systematically modified the dihedral angles about the peptide, S-NO, N-C, C-C and C-S bonds (see Figure 1) to generate a total number of 108 physically acceptable "input conformers". Specifically, we have considered: $i$ ) the two possible conformations (syn and anti) associated with the peptide bond; ii) the two possible conformations (syn and anti) about the S-NO bond; iii) the three possible staggered conformations about the N-C, C-C and C-S bonds (see Figure 2). Each "input conformer" has been subsequently fully optimized and the resulting minimum-energy structures have been properly analyzed. This has led to the identification of $n_{c}$ (86 and 43 for SNAP and NACNO, respectively) "unique conformers" that have been later subdivided into the following four groups according to the conformation about the peptide and S-NO bonds, respectively: i) synsyn, ii) syn-anti, iii) anti-syn and iv) anti-anti. 


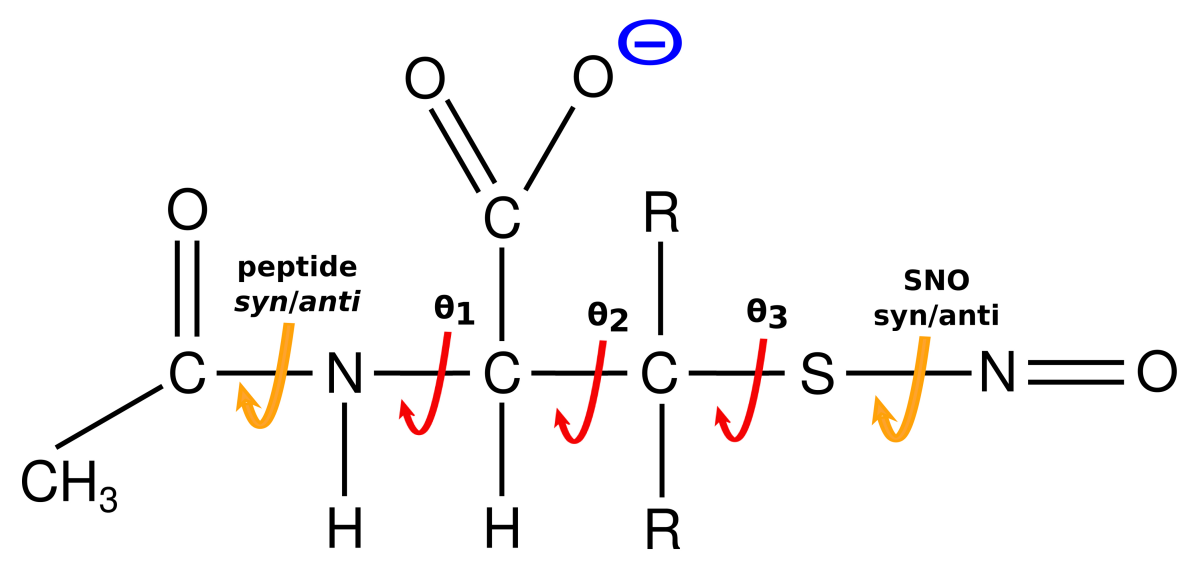

Figure 1. Rotatable bonds of SNAP $\left(\mathrm{R}=\mathrm{CH}_{3}\right)$ and $\mathrm{NACNO}(\mathrm{R}=\mathrm{H})$ about which the dihedral angles have been modified to obtain the initial 108 "input conformers" for the conformational sampling.
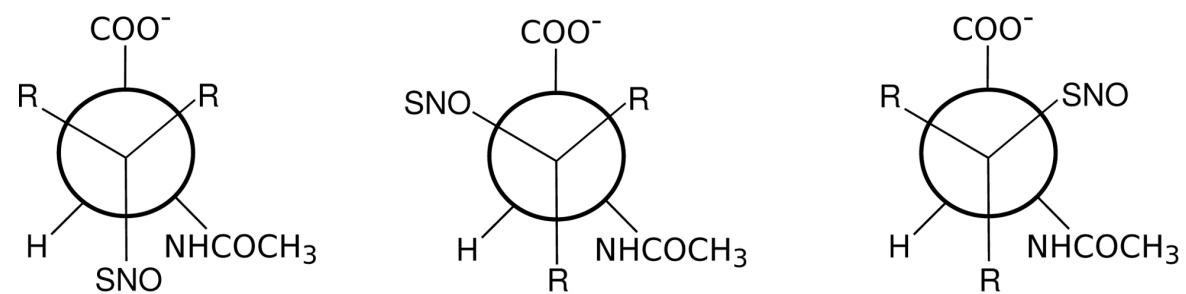

Figure 2. Definition of the three staggered conformers about the $\mathrm{C}-\mathrm{C}$ bond in SNAP $\left(\mathrm{R}=\mathrm{CH}_{3}\right)$ and NACNO $(\mathrm{R}=\mathrm{H})$. The definitions of the staggered conformers about the $\mathrm{N}-\mathrm{C}$ and $\mathrm{C}-\mathrm{S}$ bonds are analogous.

For each "unique conformer", the Gibbs free energy at $298.15 \mathrm{~K}$ has been computed, which has enabled to determine the minimum free energy conformation both for SNAP and NACNO. Furthermore, assuming that the equilibrium population of the different conformers follows the Boltzmann distribution, the calculation of the free energies has also allowed the determination of the fractional equilibrium population $P_{x}$ for each of the four groups mentioned above $(x=$ syn-syn, syn-anti, anti-syn and anti-anti):

$$
P_{x}=\frac{N_{x}}{N_{T}}=\frac{\sum_{i=1}^{n_{x}} e^{-\Delta G_{i} / R T}}{\sum_{i=1}^{n_{c}} e^{-\Delta G_{i} / R T}}
$$


with $P_{x}=N_{x} / N_{T}$ as the fraction of molecules having conformations $x$ for the peptide and the S-NO bonds, $n_{x}$ as the number of "unique conformers" belonging to the group $x, n_{c}$ as the total number of "unique conformers" and with $\Delta G_{i}, R$ and $T$ as defined for equation (1).

Table 4 summarizes the group populations for the two molecules. The most stable conformations in each case are drawn in Figure 3. The most striking result is that both for SNAP and for NACNO, the peptide anti conformation is practically the only one existing at equilibrium, being the fractional populations for the syn conformers really negligible. This can be explained by the fact that such a conformation allows the formation of a strong hydrogen bond between the NH hydrogen atom of the peptide group and the negatively charged carboxylate (see also below). In aqueous solution, water molecules are expected to strongly solvate the carboxylate group and such interactions might contribute to disrupt the intra-molecular hydrogen bond. Our results have been obtained using an implicit solvent model and therefore we do not account for explicit solute-solvent interactions. Nevertheless, our results suggest that the hydration energy does not compensate for the breaking of the intra-molecular hydrogen bond.

The scenario is more complicated for the conformations around the S-NO bond. In fact, while for SNAP the anti-anti conformers are largely predominant with respect to the anti-syn ones, for NACNO both types of conformations co-exist, with the anti-syn group being slightly preferred at room temperature $(298.15 \mathrm{~K})$. 
Table 4. Number of detected unique conformers $(\mathrm{N}), \Delta \mathrm{G}$ range $\left(\left[\Delta G_{\min }, \Delta G_{\max }\right]\right.$ in $\mathrm{kcal} \cdot \mathrm{mol}^{-1}$, relative to the most stable conformation of the molecule) and fractional equilibrium population $\left(\mathrm{P}_{\mathrm{x}}\right.$, in $\%)$ at $298.15 \mathrm{~K}$ of the syn-syn, syn-anti, anti-syn and anti-anti groups for SNAP and NACNO (the groups refer to the conformations about the peptide and the S-NO bonds, see text). The reported values have been obtained performing B3PW91/6-311+G(d,p) calculations in implicit water.

\begin{tabular}{|c|c|c|c|c|c|c|}
\hline \multirow{2}{*}{$\begin{array}{l}\text { Conformations } \\
\text { Peptide-SNO }\end{array}$} & \multicolumn{3}{|c|}{ SNAP } & \multicolumn{3}{|c|}{ NACNO } \\
\hline & $\mathrm{N}$ & {$\left[\Delta G_{\min }, \Delta G_{\max }\right]$} & $P_{x}$ & $\mathrm{~N}$ & {$\left[\Delta G_{\min }, \Delta G_{\max }\right]$} & $\mathrm{P}_{\mathrm{x}}$ \\
\hline syn-syn & 13 & [4.8-14.4] & 0.01 & 12 & [3.5-11.5] & 0.27 \\
\hline syn-anti & 20 & {$[3.2-11.0]$} & 0.30 & 10 & {$[3.7-11.7]$} & 0.15 \\
\hline anti-syn & 26 & {$[1.5-12.2]$} & 6.94 & 10 & {$[0.0-7.1]$} & 59.48 \\
\hline anti-anti & 27 & {$[0.0-9.9]$} & 92.75 & 11 & {$[0.2-8.4]$} & 40.11 \\
\hline
\end{tabular}

In order to further analyze the conformational features of SNAP and NACNO (in particular the conformational preferences about the S-NO bond), we now examine in detail the network of intra-molecular interactions of both of the two molecules using the NCI method. Considering SNAP, the networks of intra-molecular interactions for the anti-syn and the antianti conformations, which are shown in Figure 3, display similar features. In particular, the hydrogen-bond interaction between the carboxylic group and the NH hydrogen atom is evident in both cases. The difference between the anti-syn and anti-anti conformations only concerns the SNO interaction with $\mathrm{H}$ atoms. In the former case, the SNO oxygen atom establishes weak non-covalent interactions with the neighboring methyl group and with the hydrogen bound to the asymmetric carbon- $\beta$ (see green NCI surfaces in Figure 3). In contrast, in the case of the anti-anti conformation, it is the nitrogen atom that is involved in an analogous interaction-network. The preference for an SNO anti conformation in SNAP is consistent with the same preference in the case of another system characterized by a tertiary 
carbon- $\alpha$ atom with respect to the S-NO group, namely the isolated $\left(\mathrm{CH}_{3}\right)_{3} \mathrm{CSNO}$ molecule, as already shown above and also discussed by other authors ${ }^{7,8,22}$. Intuitively, one could think that this trend is due to the steric interactions between the oxygen atom and the bulky $\mathrm{R}$ group. Nevertheless, if on the one hand, the NCI results suggest that the intra-molecular van der Waals interactions between the S-NO and the methyl groups in SNAP are not crucial to rationalize the preference for the S-NO anti conformation, on the other hand, the NCI analyses do not even show the presence of non-bonding steric repulsions. One could then argue that, in absence of other specific interactions, the anti conformation is the one naturally adopted by RSNO compounds simply because this configuration minimizes the repulsive interaction between the lone-pairs of the sulfur and nitrogen atoms, which are in antiperiplanar position.

On the contrary, in case of primary carbon- $\alpha$ atoms, such as in NACNO, a specific interaction favoring the syn conformation is actually predicted. As shown in Figure 3, in the NACNO anti-syn conformer, the oxygen atom of the S-NO group is involved in a hydrogen bond-like interaction with a hydrogen atom linked to the carbon- $\alpha$. The $\mathrm{C}-\mathrm{S}-\mathrm{N}=\mathrm{O} \cdot \cdot \mathrm{H}$ pattern lies in a favorable (roughly planar) 5-membered ring configuration. In the case of the anti conformer, one could expect a comparable interaction involving the $\mathrm{N}$ atom and a four-membered ring structure but the NCI results do not reveal any significant contribution (see Figure 3, NACNO anti-anti conformer). Hence, in this situation, the syn conformation is the preferred one. For completeness, one should also notice that in NACNO, like in SNAP, the stabilizing interaction between the carboxylate group and the $\mathrm{NH}$ hydrogen atom is present. In particular, due to a less constrained molecular arrangement, which is characterized by an optimum geometry with the peptide and the carboxylate groups being roughly co-planar, this interaction is probably larger than in SNAP and, as we will show below, it will play an important role in the stability of NACNO. 


\section{SNAP anti-syn}
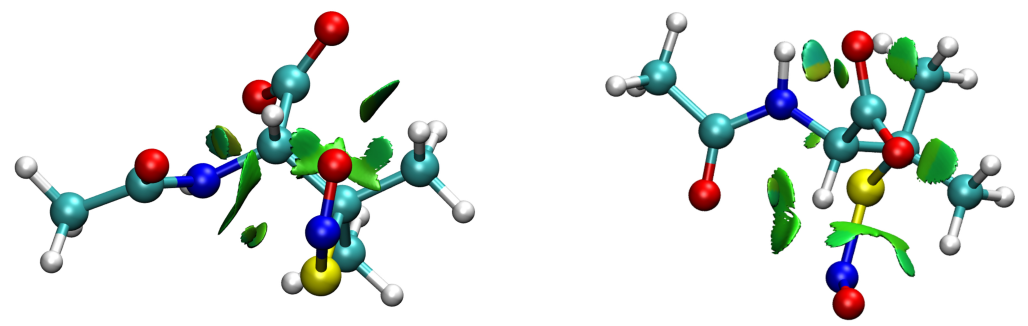

\section{SNAP anti-anti}
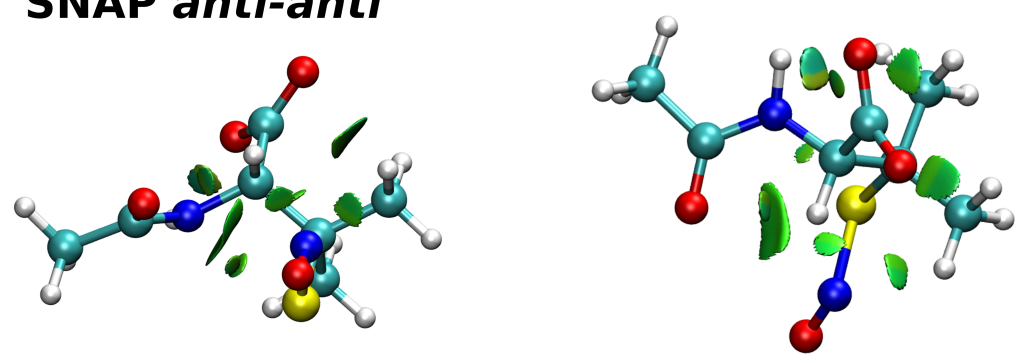

\section{NACNO anti-syn}
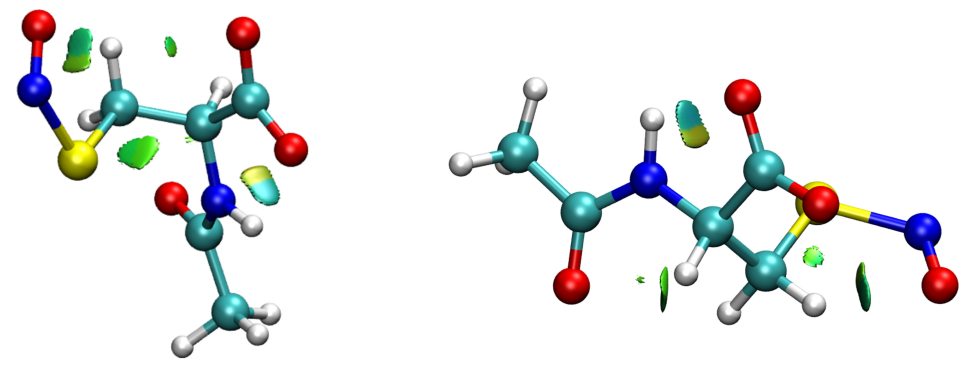

\section{NACNO anti-anti}
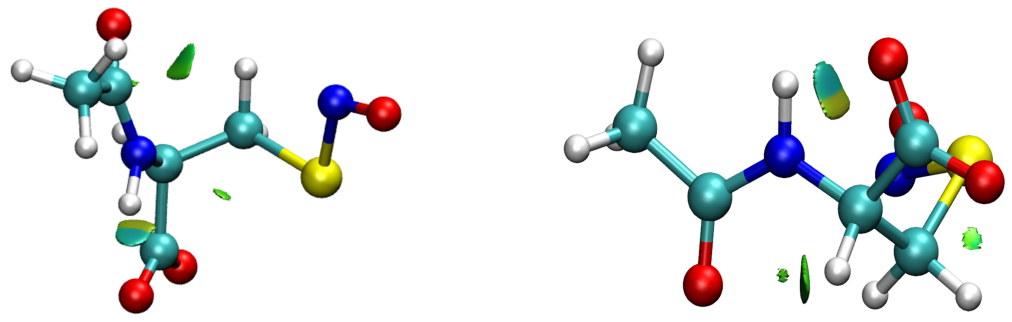

Figure 3. Most stable conformations (in two different orientations) for the SNAP and NACNO molecules and relative Non-Covalent Interactions (NCI) plots at the B3PW91/6-311+G(d,p) level. The NCI surfaces correspond to reduced density gradient values of 0.35 a.u. and they are colored on a blue-green-red scale: blue indicates strong attractive interactions, green indicates van der Waals interactions and red indicates strong steric repulsions. 
Now, let us examine the energetics for the homolytic S-NO bond dissociation of SNAP and NACNO in aqueous solution. To this aim, a conformational analysis of the RS radicals associated with these molecules has also been carried out. We started from the $n_{c}$ radical geometries obtained by removing the $\mathrm{NO} \bullet$ subunit from the structures of the $n_{c}$ "unique conformers" for the corresponding RSNO molecule. Each "input conformation" has been optimized and the obtained minimum geometries have been analyzed enabling to detect the "unique conformers" for the examined radicals. The Gibbs free energy at $298.15 \mathrm{~K}$ has been calculated for each detected conformation and the NCI analysis has been performed on the most stable conformers (see Figure 4). Finally, always considering the most stable conformers of both the undissociated molecules and the corresponding RS • radicals, we have obtained an estimation for the homolytic bond dissociation free energy for SNAP and NACNO equal to 12.8 and $17.8 \mathrm{kcal} \cdot \mathrm{mol}^{-1}$, respectively (assuming as before a $1 \mathrm{M}$ reference state in solution).

The calculations predict a significant lower free energy of dissociation in comparison with the model systems $\mathrm{CH}_{3} \mathrm{SNO}$ and $\left(\mathrm{CH}_{3}\right)_{3} \mathrm{CSNO}$ (20.7 and $18.8 \mathrm{kcal} \cdot \mathrm{mol}^{-1}$, respectively). This result can be explained by a favorable and stabilizing intra-molecular interaction occurring in the denitrosated radicals of SNAP and NACNO, namely an interaction between the charged carboxylate group and the singly occupied orbital on the $\mathrm{S}$ atom. This chalcogen-chalcogen interaction is clearly shown by the NCI analysis (see Figure 4) that displays a light-blue surface (strong attractive interaction) between the sulfur and the oxygen atoms. Moreover, Table 5 gathers some other electronic quantities that confirm this interaction, namely, the existence of a bond critical point along the $\mathrm{S} \cdots \mathrm{O}$ axis with a positive Laplacian of the electron density (density depletion) and the presence of a significant electron spin density on the oxygen atom, indicating a net charge transfer from the closed-shell of the carboxylate to the open-shell on the sulfur atom. As a result, a significant part of the spin electron density in the radicals (slightly less than $20 \%$ ) is delocalized on the carboxylate $\mathrm{O}$ atom. Interestingly, the 
effect is larger in the case of SNAP and this molecule consistently exhibits lower dissociation energy.

\section{Denitrosated SNAP radical}
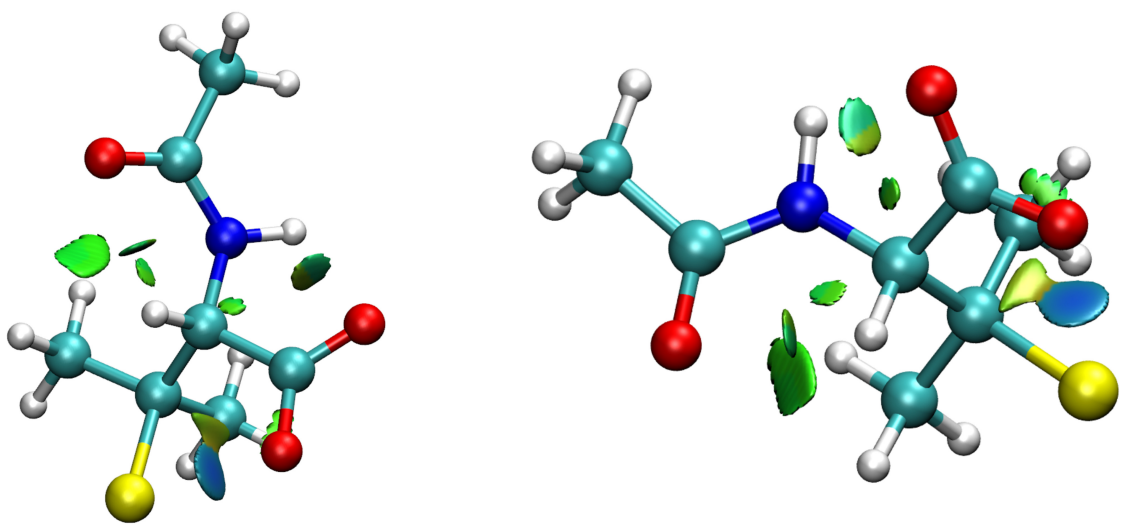

\section{Denitrosated NACNO radical}
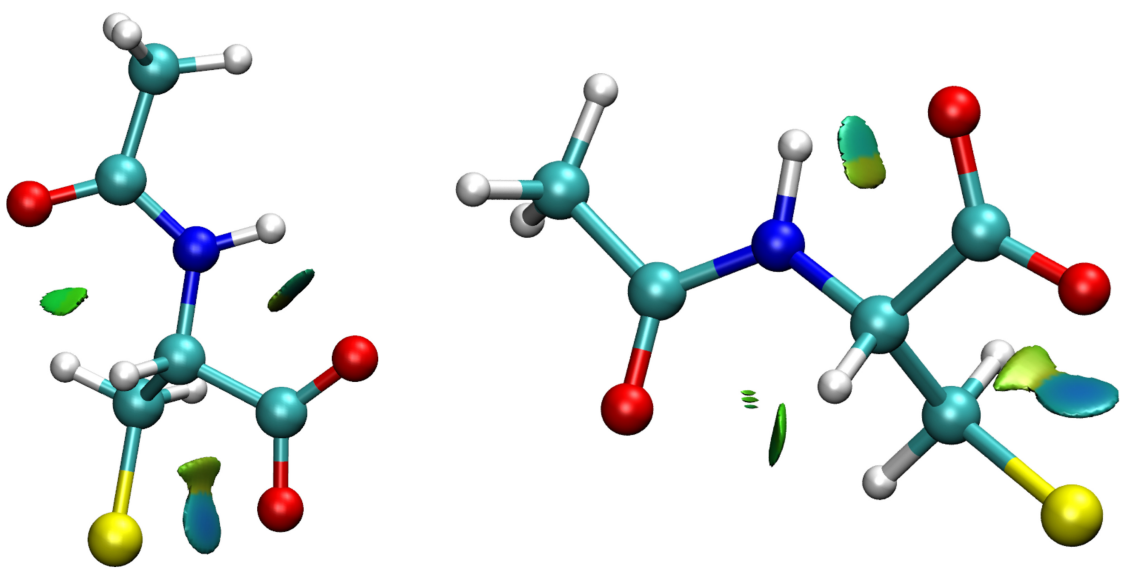

Figure 4. Most stable conformations for the denitrosated SNAP and NACNO radicals. Two different molecular orientations are depicted for each radical to better visualize all the intra-molecular interactions. The relative NCI (Non-Covalent Interactions) plots at the B3PW91/6-311+G(d,p) level are also depicted (for details on the NCI surfaces see Figure 3). 
Table 5. Calculated net integrated atomic charges $(q)$ and spin densities $(\delta)$ for the sulfur and oxygen atoms involved in the $\mathrm{S} \cdots \mathrm{O}$ intra-molecular interaction occurring in the denitrosated SNAP and NACNO radicals. The values of the electron density $\left(\rho_{B C P}\right)$ and of the its Laplacian $\left(\nabla^{2} \rho_{B C P}\right)$ at the bond critical point associated with the interaction are also shown. All the reported values are expressed in a.u..

\begin{tabular}{lcccccc}
\hline RS• & $q_{\mathrm{O}}$ & $q_{S}$ & $\delta_{O}$ & $\delta_{S}$ & $\rho_{B C P}$ & $\nabla^{2} \rho_{B C P}$ \\
\hline SNAP & -1.148 & -0.220 & 0.196 & 0.749 & 0.0344 & 0.0925 \\
NACNO & -1.159 & -0.184 & 0.176 & 0.769 & 0.0305 & 0.0817 \\
\hline
\end{tabular}

The role of the methyl groups in SNAP deserves some comments since, as already pointed out above, the lower stability of SNAP with respect to NACNO contrasts with the usual larger stability of tertiary RSNOs. ${ }^{6}$ In principle, two main effects can be considered. The first one is electronic and it can be associated with the usual "inductive effect" of alkyl groups, and/or with the increase of the molecular polarizability upon alkylation. The second effect is an indirect one. It results from the modifications of the molecular geometry upon substitution of the hydrogen atoms in NACNO by the more bulky methyl groups in SNAP. We have carried out some calculations to assess these two different effects. From the results, it appears that the joint effects of induction and polarization play a minor role. For instance, the substitution of the methyl groups in SNAP by hydrogen atoms, keeping the rest of the atoms at the same geometry, does not modify the chalcogen-chalcogen interaction strength significantly. In fact, the $\mathrm{S}$ and $\mathrm{O}$ spin densities are 0.748 and 0.200 , respectively, very close to those in SNAP. Besides, calculations for the electron affinity in the primary and model radicals $\left(\mathrm{CH}_{3}\right) \mathrm{H}_{2} \mathrm{CS}$ • and $\left(\mathrm{CH}_{3}\right)_{3} \mathrm{CS} \bullet$ reveal only minor differences and, in addition, the largest affinity (in absolute value) corresponds to the least substituted radical (-106.1 and $-105.7 \mathrm{kcal} / \mathrm{mol}$ for $\left(\mathrm{CH}_{3}\right) \mathrm{H}_{2} \mathrm{CS} \bullet$ and $\left(\mathrm{CH}_{3}\right)_{3} \mathrm{CS} \bullet$ respectively). Therefore, we conclude that indirect effects due to the structural changes in SNAP with respect to NACNO must be considered as the main 
reason for the different strength of the chalcogen-chalcogen interaction in the two different cases.

It is worth reminding that the calculated solvation effects for the model RSNO molecules reported above are negligible (see Table 3). In the case of SNAP and NACNO, the situation might be different because these systems are charged and the involved solvation energies are much larger. However, the analysis of the solvation free energies (see Table S1 in the Supporting Information) shows that, both for SNAP and NACNO, they do not play a noticeable role in the energetics of the dissociation process. In fact, the total stabilization of the radical products is not far from the stabilization of the reactant: in the case of NACNO the effect is really negligible, while, in the case of SNAP, solvation tends to favor the dissociation by $0.7 \mathrm{kcal} \cdot \mathrm{mol}^{-1}$.

Finally, it is easy to note that the computed dissociation energies of SNAP and NACNO in aqueous solution display a significant difference (about $5 \mathrm{kcal} \cdot \mathrm{mol}^{-1}$ ), with a larger value for NACNO. If on the one hand this difference stems from the larger stabilization of the NACNO reactant through the contribution of two main intra-molecular hydrogen bonds, on the other hand it also derives from a larger stabilization of the denitrosated SNAP radical due to a chacogen-chalcogen interaction. As discussed in the introduction, the RSNOs stability is still a matter of debate because the mechanisms of dissociation have not been completely understood yet. However, the greater homolytic dissociation energy of the S-NO bond in NACNO suggests that this molecule should exhibit a larger stability than SNAP in aqueous solution, as experimentally observed. Furthermore, the computed free energy difference, 5 $\mathrm{kcal} \cdot \mathrm{mol}^{-1}$, is consistent with the relative value of measured half-life times ${ }^{3}(500 \mathrm{~h}$ and $2 \mathrm{~h}$ for NACNO and SNAP, respectively), which have been indeed obtained through experiments that assumed a first-order kinetics $\left(\mathrm{t}_{1 / 2}=\ln 2 / \mathrm{K}\right)$ so that the free energy for the activation processes should differ by about $3.3 \mathrm{kcal} \cdot \mathrm{mol}^{-1}$. 


\section{Conclusions}

In this paper, we have examined the relationship between the structure and stability of the RSNO molecules SNAP ( $S$-nitroso- $N$-acetylpenicillamine) and NACNO ( $S$-nitroso- $N$ acetylcysteine), whose pharmacological relevance has been already proved experimentally. These molecules display significant differences in half-life times in aqueous solution despite their very similar structures, which actually differs only by the number of methyl groups on the carbon- $\alpha$ bound to the S-NO function. In addition, and in contrast to the usual larger stability of tertiary RSNOs with respect to primary ones, ${ }^{6}$ SNAP (a tertiary derivative) has been found to be less stable than NACNO (a primary derivative). ${ }^{3}$

A thorough study of their conformational preferences has shown that, while for the peptide bond moiety the only existing conformation at $298.15 \mathrm{~K}$ is practically the anti one in both cases, the equilibrium distribution of the conformers about the S-NO bond depends on the molecule. In contrast to previous statements, the ratio between the syn and the anti conformers is not directly related to the steric effect of the methyl groups on the carbon- $\alpha$. Rather, our investigations have shown that the value of this ratio results from the balance of opposing interactions in the syn conformation, namely, the repulsive interaction between the sulfur and the nitrogen lone pairs and the stabilizing contribution of a $\mathrm{C}(\alpha)-\mathrm{H} \cdots \mathrm{O}$ hydrogenbond. Of course, in the tertiary derivative SNAP, the second interaction is not possible, and the anti conformation predominates. In NACNO, both the interactions are present and almost compensate each other, so that both the anti and the syn conformations coexist, with a slight preference for the syn one. This interpretation is supported by a theoretical analysis of the non-covalent interactions based on the topology of the electron density and of its corresponding reduced gradient.

Furthermore, through the calculation of homolytic Bond Dissociation Free Energies, our studies have shown i) lower dissociation energies of SNAP and NACNO compared to the 
model RSNO compounds $\left(\mathrm{R}=\mathrm{CH}_{3}\right.$, $\left.\mathrm{tBu}\right)$ as well as ii) a higher stability of NACNO over SNAP, in agreement with the experimentally determined half-life times of the two species. The analysis of the electron density reveals that the radicals of these two molecules are strongly stabilized by a chalcogen-chalcogen type interaction involving a carboxylate $\mathrm{O}$ atom and the S• lone pair center, with some charge transfer and delocalization of the spin density. This interaction is stronger in SNAP and decreases its S-NO dissociation energy to a larger extent than in NACNO.

In light of the obtained results, we envisage the study of larger and possibly pharmacologically relevant RSNO molecules (e.g., the recently proposed $S, S^{\prime}$ dinitrosobucillamine ${ }^{42}$ ), which will allow us to shed further light on the role played by the intra-molecular interactions in the stabilization of the RSNOs. Furthermore, in order to better establish the influence of the solvation effects and of the biological environment, we are also planning to perform Molecular Dynamics simulations and new Quantum Mechanics/Molecular Mechanics (QM/MM) computations with the explicit inclusion of water molecules and/or protein residues. The main goal of all these studies is to get more and more insights into the RSNOs structure, stability and reactivity, which will hopefully lead us to propose new $S$-nitrosothiols characterized by an enhanced pharmacological potency. In particular, we believe that a detailed analysis of the inter- and intra-molecular interactions in which the different RSNOs are involved will be of fundamental importance to propose suitable pharmacophore descriptors that will drive the design of new potential drugs.

\section{Supporting Information Available}

Details of the NCI calculations. Figures S1-S2: Reduced Density Gradient plots for SNAP, NACNO and their corresponding denitrosated radicals in different conformations. Table S1: Free energies of solvation and solvation effects on the S-NO dissociation processes of SNAP 
and NACNO at $298.15 \mathrm{~K}$. XYZ coordinates for the most stable syn-syn, syn-anti, anti-syn and anti-anti conformers of SNAP and NACNO and XYZ coordinates for the most stable conformers of the denitrosated SNAP and NACNO radicals. This material is available free of charge via the Internet at http://pubs.acs.org/.

\section{References}

1. Münzel, T.; Daiber, A.; Gori, T. Nitrate therapy : new aspects concerning molecular action and tolerance. Circulation 2011, 123, 2132-2144.

2. Widlansky, M. E.; Gokce, N.; Keaney, J. F., Jr.; Vita, J. A. The clinical implications of endothelial dysfunction. J. Am. Coll. Cardiol. 2003, 42, 1149-1160.

3. Gaucher, C.; Boudier, A.; Dahboul, F.; Parent, M.; Leroy, P. Snitrosation/denitrosation in cardiovascular pathologies: facts and concepts for the rational design of S-nitrosothiols. Curr Pharm Des. 2013, 19, 458-472.

4. Parent, M.; Dahboul, F.; Schneider, R.; Clarot, I.; Maincent, P.; Leroy, P.; Boudier, A. A complete physicochemical identity card of S-nitrosoglutathione. Curr. Pharm. Anal. 2013, 9, 31-42.

5. Xiao-Hong, L.; Zheng-Xin, T.; Xian-Zhou, Z. Natural bond orbital analysis of some S-nitrosothiols biological molecules. Int. J. Quantum Chem. 2010, 110, 1565-572.

6. Roy, B.; Moulinet d'Hardemare, A.; Fontecave, M. New thionitrites: synthesis, stability, and nitric oxide generation. J. Org. Chem. 1994, 59, 7019-7026.

7. Bartberger, M. D.; Houk, K. N.; Powell, S. C.; Mannion, J. D.; Lo, K. Y.; Stamler, J. S.; Toone, E. J. Theory, Spectroscopy, and Crystallographic Analysis of $S$ Nitrosothiols: Conformational Distribution Dictates Spectroscopic Behavior. J. Am. Chem. Soc. 2000, 122, 5889-5890. 
8. Bartberger, M. D.; Mannion, J. D.; Powell, S. C.; Stamler, J. S.; Houk, K. N.; Toone, E. J. J. Am. Chem. Soc. 2001, 123, 8868-8869.

9. Lü, J.-M.; Wittbrodt, J. M.; Wang, K.; Wen, Z.; Schlegel, H. B.; Wang, P. G.; Cheng, J.-P. NO Affinities of $S$-Nitrosothiols: A Direct Experimental and Computational Investigation of RS-NO Bond Dissociation Energies. J. Am. Chem. Soc. 2001, 123, 2903-2904.

10. Baciu, C.; Gauld, J. W. An Assessment of Theoretical Methods for the Calculation of Accurate Structures and SN Bond Dissociation Energies of $S$-Nitrosothiols (RSNOs). J. Phys. Chem. A 2003, 107, 9946-9952.

11. Fu, Y.; Mou, Y.; Lin, Liu, L.; Guo, Q.-X. Structures of the $\mathrm{X}-\mathrm{Y}-\mathrm{NO}$ Molecules and Homolytic Dissociation Energies of the $\mathrm{Y}-\mathrm{NO}$ Bonds $(\mathrm{Y}=\mathrm{C}, \mathrm{N}, \mathrm{O}, \mathrm{S})$. J. Phys. Chem. A 2002, 106, 12386-12392.

12. Timerghazin, Q. K.; Peslherbe, G. H.; English, A. M. Resonance Description of $S$ Nitrosothiols: Insights into Reactivity. Org. Lett. 2007, 9, 3049-3052..

13. Timerghazin, Q. K.; English, A. M.; Peslherbe, G. H. On the multireference character of S-nitrosothiols: A theoretical study of HSNO. Chem. Phys. Lett. 2008, 454, 24-29.

14. Flister, M.; Timerghazin, Q. K. Structure, Stability, and Substituent Effects in Aromatic $S$-Nitrosothiols: The Crucial Effect of a Cascading Negative Hyperconjugation/Conjugation Interaction. J. Phys. Chem. A 2014, 118, 9914-9924.

15. Li, X.-H.; Cui, H.-L.; Zhang, X.-Z. PCM Study of Bond Dissociation Energies of the S-NO Bond: A DFT Study. J. Sol. Chem. 2013, 42, 1221-1228.

16. Talipov, M. R.; Timerghazin, Q. K. Protein Control of $S$-Nitrosothiol Reactivity: Interplay of Antagonistic Resonance Structures. J. Phys. Chem. B 2013, 117, 18271837. 
17. Timerghazin, Q. K.; Talipov, M. R. Unprecedented External Electric Field Effects on S-Nitrosothiols: Possible Mechanism of Biological Regulation ? J. Phys.Chem. Lett. 2013, 4, 1034-1038.

18. Liang, J.; Cheng, S.; Hou, J.; Xu, Z.; Zhao, Y.-L. Car-Parinello molecular dynamics simulations of thionitroxide and S-nitrosothiol in the gas phase, methanol, and water-A theoretical study of S-nitrosylation. Sci. China Chem. 2012, 55, 2081-2088.

19. Zhao, Y.-L.; McCarren, P. R.; Houk, K. N.; Choi, B. Y.; Toone, E. J. NitrosoniumCatalyzed Decomposition of $S$-Nitrosothiols in Solution: A Theoretical and Experimental Study. J. Am. Chem. Soc. 2005, 127, 10917-10924.

20. Moran, E. E.; Timerghazin, Q. K.; Kwong, E.; English, A. M. Kinetics and Mechanism of $S$-Nitrosothiol Acid-Catalyzed Hydrolysis: Sulfur Activation Promotes Facile NO ${ }^{+}$Release. J. Phys. Chem. B 2011, 115, 3112-3126.

21. Fernández-González, M. Á.; Marazzi, M.; López-Delgado, A.; Zapata, F.; GarcíaIriepa, C.; Rivero, D.; Castaño O.; Temprado M.; Frutos, L. M. Structural Substituent Effect in the Excitation Energy of a Chromophore: Quantitative Determination and Application to S-Nitrosothiols. J. Chem. Theory Comput. 2012, 8, 3293-3302.

22. Marazzi, M.; López-Delgado, A.; Fernández-González, M. A.; Castaño, O.; Frutos, L. M.; Temprado, M. Modulating Nitric Oxide Release by $S$-Nitrosothiol Photocleavage: Mechanism and Substituent Effects. J. Phys. Chem. A. 2012, 116, 7039-7049.

23. Ivanova, L. V.; Anton, B. J.; Timerghazin, Q. K. On the possible biological relevance of HSNO isomers: a computational investigation. Phys. Chem. Chem. Phys. 2014, 16, $8476-8486$

24. Talipov, M. R.; Khomyakov, D. G.; Xian, M.; Timerghazin, Q. K. Computational design of $S$-nitrosothiol “click” reactions. J. Comp. Chem. 2013, 34, 1527-1530. 
25. Timerghazin, Q. K.; Peslherbe, G. H.; English, A. M. Structure and stability of HSNO, the simplest S-nitrosothiol. Phys. Chem. Chem. Phys. 2008, 10, 1532.

26. Canneva, A.; Erben, M. F.; Romano, R. M.; Vishnevskiy, Y. V.; Reuter, C. G.; Mitzel, N. W.; Della Vedova, C. O. The Structure and Conformation of $\left(\mathrm{CH}_{3}\right)_{3}$ CSNO. Chem. Eur. J. 2015, 21, 10436-10442.

27. Frisch, M. J.; Trucks, G. W.; Schlegel, H. B.; Scuseria, G. E.; Robb, M. A.; Cheeseman, J. R.; Scalmani, G.; Barone, V.; Mennucci, B.; Petersson, G. A.; et al. Gaussian 09, Revision D.01, Gaussian: Wallingford CT, USA, 2009.

28. Becke, A. D. Density-functional exchange-energy approximation with correct asymptotic behavior. Phys. Rev. A 1988, 38, 3098-3100.

29. Lee, C.; Yang, W.; Parr, R. G. Development of the Colle-Salvetti correlation-energy formula into a functional of the electron density. Phys. Rev. B 1988, 37, 785-789.

30. Perdew, J. P.; Burke, K.; Ernzerhof, M. Generalized gradient approximation made simple. Phys. Rev. Lett. 1996, 77, 3865-3868.

31. Perdew, J. P. Density-functional approximation for the correlation energy of the inhomogeneous electron gas. Phys. Rev. B 1986, 33, 8822-8824.

32. Perdew, J. P.; Wang, Y. Accurate and simple analytic representation of the electrongas correlation energy. Phys. Rev. B 1992, 45, 13244-13250.

33. Cramer, C. J.; Truhlar, D. G. Implicit Solvation Models: Equilibria, Structure, Spectra, and Dynamics. Chem. Rev. 1999, 99, 2161-2200.

34. Curutchet, C.; Cramer, C. J.; Truhlar, D. G.; Manuel, F.; Rinaldi, D.; Orozco, M.; Luque, F. J. Electrostatic component of solvation: comparison of SCRF continuum models. J. Comput. Chem. 2003, 24, 284-297.

35. Tomasi, J.; Mennucci, B.; Cammi, R. Quantum Mechanical Continuum Solvation Models. Chem. Rev. 2005, 105, 2999-3094. 
36. Cancès, E.; Mennucci, B.; Tomasi, J. A new integral equation formalism for the polarizable continuum model: Theoretical background and applications to isotropic and anisotropic dielectrics. J. Chem. Phys. 1997, 107, 3032-3041.

37. Kelly, C. P.; Cramer, C. J.; Truhlar, D. G. Aqueous Solvation Free Energies of Ions and Ion-Water Clusters Based on an Accurate Value for the Absolute Aqueous Solvation Free Energy of the Proton. J. Phys. Chem. B. 2006, 110, 16066-16081.

38. Johnson, E. R.; Keinan, S.; Mori-Sánchez, P.; Contreras-García, J.; Cohen, A. J.; Yang, W. Revealing Noncovalent Interactions. J. Am. Chem. Soc. 2010, 132, 64986506

39. Contreras-García, J.; Johnson, E. R.; Keinan, S.; Chaudret, R.; Piquemal, J.-P.; Beratan, D. N.; Yang, W. NCIPLOT: A Program for Plotting Noncovalent Interaction Regions. J. Chem. Theory Comput. 2011, 7, 625-632.

40. Bader, R. F. W. Atoms in Molecules: A Quantum Theory, Oxford University Press: Oxford, U.K., 1990.

41. Keith, T. A. AIMAll, Version 13.11.04; TK Gristmill Software: Overland Park, KS, USA, 2012; available online: aim.tkgristmill.com.

42. Dahboul, F.; Perrin-Sarrado, C.; Boudier, A.; Lartaud, I.; Schneider, R.; Leroy, P. $\mathrm{S}, \mathrm{S}^{\prime}$-dinitrosobucillamine, a new nitric oxide donor, induces a better vasorelaxation than other S-nitrosothiols. Eur. J. Pharmacol. 2014, 730, 171-179. 
TOC Graphic

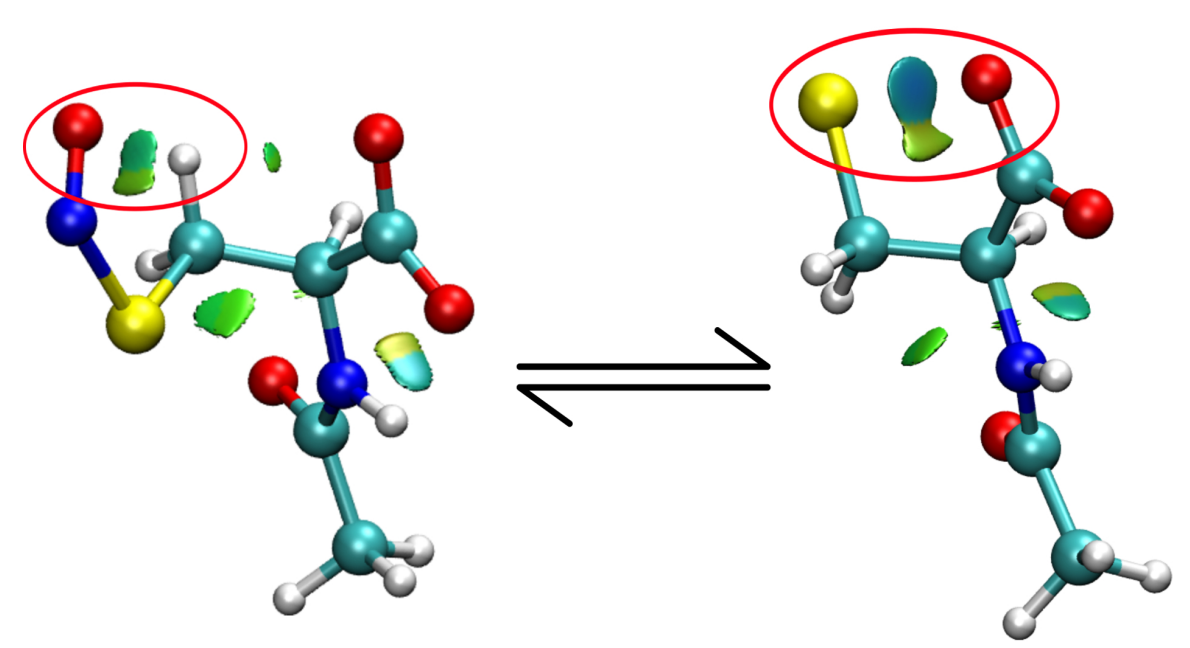

